\title{
Congestive hepatic fibrosis score: a novel histologic assessment of clinical severity
}

\author{
Dao-Fu Dai ${ }^{1}$, Paul E Swanson ${ }^{1}$, Eric V Krieger ${ }^{2}$, Iris W Liou ${ }^{2}$, Robert L Carithers ${ }^{2}$ and \\ Matthew M Yeh ${ }^{1,2}$ \\ ${ }^{1}$ Department of Pathology, University of Washington School of Medicine, Seattle, WA, USA and ${ }^{2}$ Department \\ of Medicine, University of Washington School of Medicine, Seattle, WA, USA
}

\begin{abstract}
Chronic right heart failure predisposes to hepatic passive congestion and centrizonal necrosis that may lead to hepatic fibrosis (cardiac sclerosis). Although there have been several studies on the histologic features of congestive hepatopathy, there is no available grading system. In this study we developed a novel grading system for congestive hepatic fibrosis. Liver biopsies were examined in patients with chronic heart failure of various etiologies including congenital heart disease, idiopathic cardiomyopathy, ischemic heart disease, and valvular heart disease. The cases with available echocardiography and/or right heart catheterization were included. Cases with other types of underlying chronic liver diseases, alcoholic liver disease, significant steatosis ( $>20 \%$ ), malignant neoplasm, and acute heart failure or shock were excluded. After exclusion, 42 cases were included in the study. We herein proposed a novel congestive hepatic fibrosis score and correlated it with the right heart structure and function obtained by echocardiography and/or right heart catheterization. Our results showed that congestive hepatic fibrosis score is well correlated with the right atrial pressure $(P$ for trend $<\mathbf{0 . 0 0 1 )}$ ). The presence of portal fibrosis (congestive hepatic fibrosis scores 2 and 3 ) is associated with significantly higher right atrial pressure than those with no fibrosis $(P<0.001)$ or with centrizonal fibrosis only $(P=0.02)$. Congestive hepatic fibrosis score is also significantly associated with increasing severity of right atrial dilatation $(P=0.03)$ and right ventricular dilatation $(P=0.02)$, indicators for chronic volume and/or pressure overload. Other histopathologic features include sinusoidal dilatation and centrizonal hepatocyte atrophy. In summary, although sinusoidal dilatation and centrizonal fibrosis are the hallmarks of hepatic passive congestion, the presence of portal fibrosis is suggestive of more advanced disease, as it correlates with more severe impairment of right heart function, regardless of the etiologies of right heart failure. Congestive hepatic fibrosis score is a useful indicator of clinical severity.
\end{abstract}

Modern Pathology (2014) 27, 1552-1558; doi:10.1038/modpathol.2014.79; published online 13 June 2014

Liver pathology in patients with congestive heart failure has been recognized for decades. The histopathology of cardiac hepatopathy or 'nutmeg liver' was initially described in liver necropsy specimens by Sherlock ${ }^{1}$ in 1951, whose salient observations included sinusoidal dilatation and centrilobular hepatic necrosis. Several studies have since confirmed and further characterized these histologic findings in post-mortem liver specimens from patients with various cardiac diseases or shock, including centrilobular congestion, with or without necrosis, inflammation, sclerosis, and regenerative

Correspondence: Professor MM Yeh, MD, PhD, Department of Pathology, University of Washington School of Medicine, 1959 NE Pacific Street, NE140D, Box 356100, Seattle, WA 98195, USA. E-mail: myeh@uw.edu

Received 5 March 2014; revised 16 March 2014; accepted 18 March 2014; published online 13 June 2014 hyperplasia. ${ }^{2-4}$ The progression to advanced-stage disease (cardiac cirrhosis), however, remains rare, despite the common manifestation of centrilobular fibrosis. ${ }^{2,5}$ In one pertinent autopsy series, centrilobular congestion was closely associated with clinical conditions that present as elevated central venous pressure, whereas centrilobular necrosis often occurred in the setting of shock. ${ }^{3}$ Although hemodynamic derangement induces cardiac hepatopathy, very few studies have correlated hemodynamic parameters with histological findings. In one such analysis, Myers et $a l^{5}$ reported that elevated right atrial pressure and hepatic venous pressure were associated with the presence of centrilobular necrosis, inflammation, and sinusoidal dilatation, but surprisingly, not with hepatic fibrosis. Because the latter observations raise concerns about the reproducibility of histopathologic observations in cardiac hepatopathy, we believe that ongoing 
investigations of this condition, including metaanalyses of existing studies, would be aided by a robust histologic scoring system.

In the current study, we propose a simplified histologic scoring system for congestive hepatopathywhat we will refer to as the congestive hepatic fibrosis score-that may serve as a clinically useful indicator of the severity of congestive hepatopathy. Our findings demonstrate that the congestive hepatic fibrosis score correlates well with echocardiographic and hemodynamic parameters.

\section{Materials and methods}

\section{Patient Selection}

We searched the University of Washington Medical Center Pathology database to identify patients with liver biopsies between 2003 and 2012 who met the specific keywords of either 'heart disease,' 'heart failure,' or 'cardiomyopathy.' The 64 patients meeting these search parameters were filtered using a specific inclusion criterion: the availability of complete echocardiographic studies within 4 months of the date of liver biopsy; and several exclusion criteria were used: (1) viral or alcoholic hepatitis, (2) transplanted liver, (3) malignancy, (4) steatosis $>20 \%$ of hepatocytes, regardless of the etiology, (5) clinical demonstration (or documented suspicion) of shock, and (6) biopsies with length $<1.5 \mathrm{~cm}$. A total of 54 patients met the inclusion criteria. Of these, 8 were excluded: 3 had viral/alcoholic hepatitis, 3 had significant steatosis, and 2 had malignancy. There were 42 patients available for final analysis.

\section{Clinical, Echocardiographic, and Cardiac Catheterization Data}

All clinical, laboratory, echocardiographic, and cardiac catheterization data analyzed in this study were obtained from electronic medical records of our institution. Echocardiographic data included left ventricular ejection fraction, echocardiographic diagnosis, estimated right atrial pressure, and the degree of right atrial and right ventricular dilatation. For cardiac catheterization, we included the mean right atrial pressure data from right heart catheterization studies performed within 4 months of the echocardiography. Concurrent liver tests included serum aspartate aminotransferase (AST), alanine aminotransferase (ALT), total alkaline phosphate (ALP), $\gamma$-glutamyl transferase (GGT), and total and direct bilirubin.

\section{Congestive Hepatic Fibrosis Score and Histopathologic Examination}

We designed a simplified congestive hepatic fibrosis score based on prior literature review ${ }^{2,3,5}$ and 9 index autopsy cases (2011-2012) that met the aforementioned inclusion and exclusion criteria. Based on the pattern of fibrosis, scores of $0,1,2 \mathrm{~A}, 2 \mathrm{~B}, 3$, and 4 may be assigned as follows: score 0 , no fibrosis; score 1, central fibrosis; score 2A, central zone and portal fibrosis, with accentuation of fibrosis in the central zone; score 2B, moderate portal fibrosis and central zone fibrosis, with accentuation of fibrosis in the portal zone; score 3, bridging fibrosis; score 4, cirrhosis (see also Table 1 and Figure 1). Figure 2 presents examples of the congestive hepatic fibrosis score using a Masson trichrome stain.

For histopathologic data collection, archived hematoxylin and eosin and Masson trichromestained sections of liver biopsy samples from each patient were evaluated.

The adequacy of the biopsy specimen is defined as biopsy length of at least $1.5 \mathrm{~cm}$. All cases that were analyzed meet these criteria. Biopsies were evaluated for sinusoidal dilatation and hepatocyte atrophy, and were assigned a consensus congestive hepatic fibrosis score by two pathologists (MMY and PES) who were blinded to clinical and outcome data.

\section{Statistical Analysis}

Continuous variables were presented using mean \pm s.e.m., and compared using the two-sample $t$-test or one-way ANOVA, followed by Sidak post hoc analysis. Categorical variables were compared using the $\chi^{2}$ test or Fisher's exact test. Nonparametric test for trend was applied to investigate the relationship between the congestive hepatic fibrosis score and the right atrial pressure. The $P$-values of $<0.05$ were considered statistically significant. All statistical analyses were performed using Stata IC version 10 (College Station, TX, USA).

\section{Results}

Liver biopsies from 42 patients with heart diseases met the inclusion and exclusion criteria and were assigned a congestive hepatic fibrosis score. The demographic data and heart disease status of our study population stratified by the congestive hepatic fibrosis score are summarized in Table 2. The mean age was $51.8 \pm 1.8$ years, with 29 of 42 patients $(69 \%)$ being male. There was no significant difference in age or gender across different congestive hepatic fibrosis score groups. Liver tests, including

Table 1 Proposed scoring system for congestive hepatic fibrosis

Congestive hepatic fibrosis score

$1 \quad$ Central zone fibrosis

2A Central zone and mild portal fibrosis, with accentuation at central zone

2B At least moderate portal fibrosis and central zone fibrosis, with accentuation at portal zone

3 Bridging fibrosis

$4 \quad$ Cirrhosis 


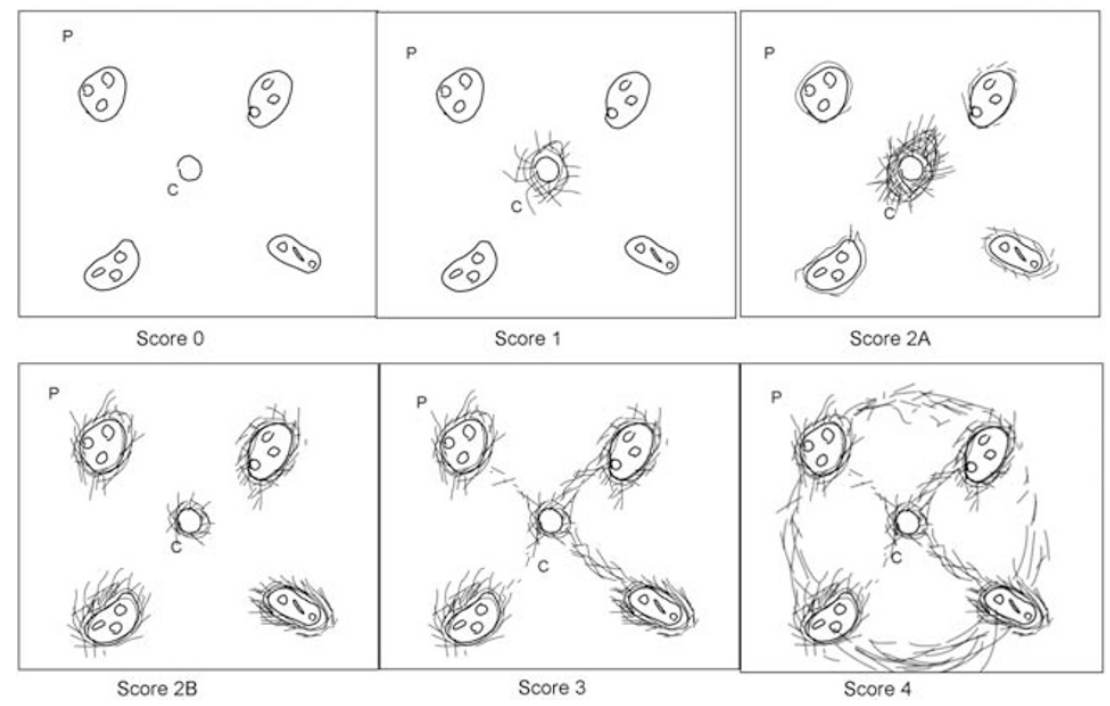

Figure 1 Diagram of the proposed congestive hepatic fibrosis score: score 0, no fibrosis; score 1, central zone fibrosis only; score 2A, central zone and portal fibrosis, with accentuation of fibrosis in the central zone; score $2 \mathrm{~B}$, moderate portal fibrosis and central zone fibrosis, with accentuation of fibrosis in the portal zone; score 3, bridging fibrosis; and score 4, cirrhosis.

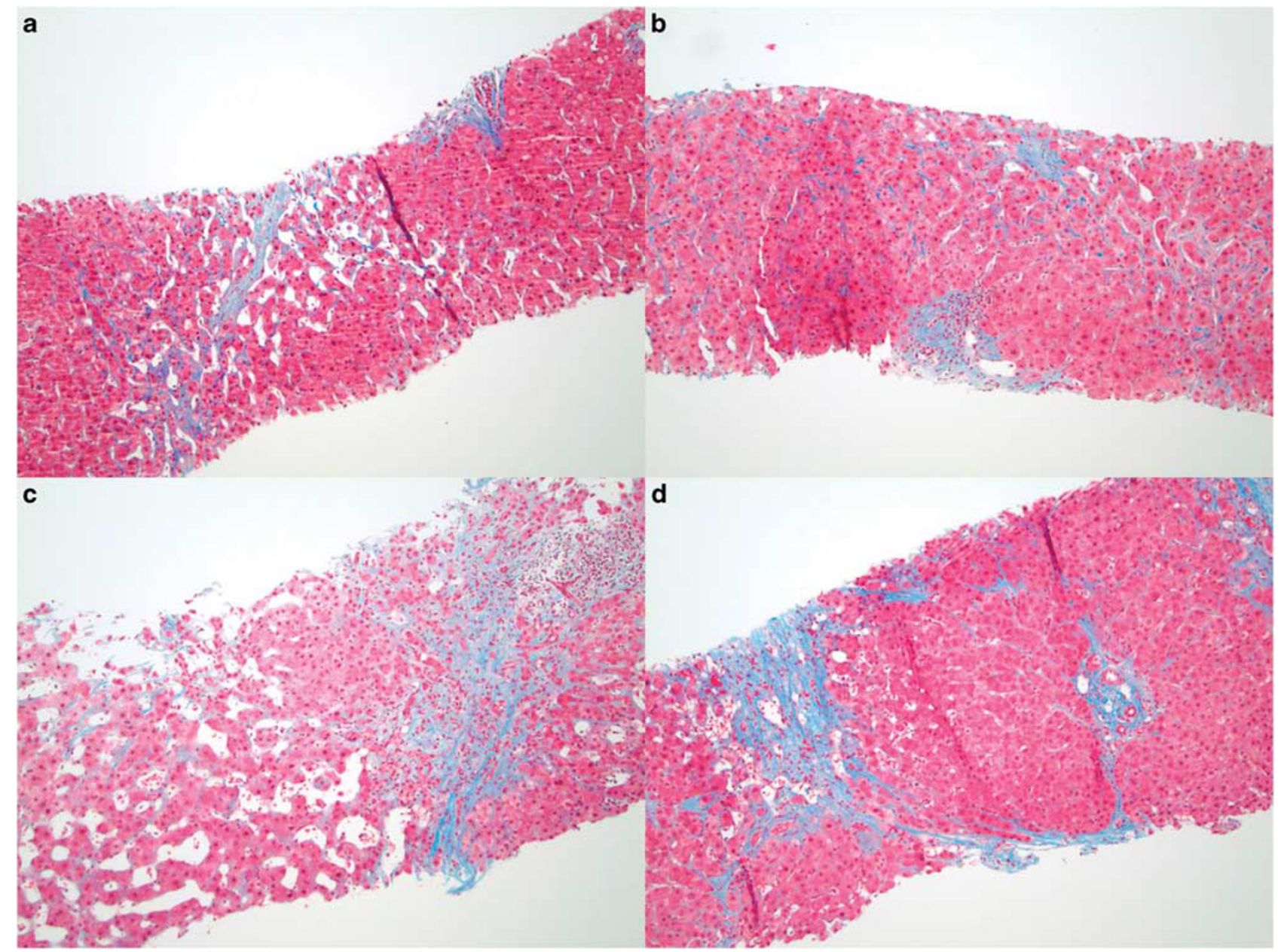

Figure 2 Examples of the congestive hepatic fibrosis score using Masson trichrome stain: (a) Centrizonal fibrosis without portal fibrosis (score 1) in a background of sinusoidal dilatation, (b) centrizonal accentuated fibrosis with mild portal fibrosis (score 2A), (c) portal accentuated fibrosis and central zone fibrosis (score 2B) and sinusoidal dilatation, and (d) bridging fibrosis (score 3). Masson trichrome stain, $\times 100$ magnification. 
serum AST, ALT, ALP, GGT, and total and direct bilirubin, were also not significantly different across congestive hepatic fibrosis score. Similarly, the indication for liver biopsy (preheart transplant evaluation, abnormal liver tests, and abnormal liver imaging (suspected cirrhosis) by ultrasound, CT, or MRI) was not significantly different across congestive hepatic fibrosis scores. The left ventricular systolic function as indicated by LV ejection fraction (overall mean LV ejection fraction of $34.5 \% \pm 3.6$; reference value: $>55 \%$ ) was not significantly different across congestive hepatic fibrosis score, nor was the primary underlying cardiac disease (Table 2; 16 patients with idiopathic cardiomyopathy, 11 with dilated cardiomyopathy, 2 with hypertrophic cardiomyopathy, 3 with restrictive cardiomyopathy, 10 with congenital heart disease-including 5 with transposition of great arteries status after corrective surgeries, 3 with Ebstein's anomaly, and 2 with tricuspid atresia -7 with coronary heart disease and ischemic cardiomyopathy, 4 with valvular heart disease, and 5 diagnosed as congestive heart failure without any specific etiology).

\section{Histologic Findings and Congestive Hepatic Fibrosis Score}

Overall, tissue preservation was adequate in all biopsies for histologic evaluation. The most striking finding was the frequency of sinusoidal dilatation and centrizonal hepatocyte atrophy in the study popu- lation (Figure 3). Of 37 patients with elevated right atrial pressure ( $>10 \mathrm{~mm} \mathrm{Hg}), 35(94.6 \%)$ had sinusoidal dilatation and $31(86.1 \%)$ had hepatocyte atrophy.

The correlation of congestive hepatic fibrosis score with other histopathologic findings is shown in Table 3. Patients with a congestive hepatic fibrosis score of at least 1 (ie, any pattern of fibrosis) had a significantly higher proportion of cases with hepatocyte atrophy ( $\sim 89 \%)$ compared with those with a congestive hepatic fibrosis score of 0 (or no fibrosis, $16 \%$ ); however, there was no incremental increase in the proportion of hepatocytes undergoing atrophy with increasing severity of congestive hepatic fibrosis score. There was a suggestion of increasing sinusoidal dilation with increasing congestive hepatic fibrosis score, although this did not reach statistical significance $(P=0.13$, Table 3$)$.

Figure 4 illustrates the correlation between congestive hepatic fibrosis score and the severity of right heart dysfunction. Increasing congestive hepatic fibrosis score is significantly associated with an increase in right atrial pressure $(P$ for trend $<0.001$, Figure 4a). Furthermore, patients with portal fibrosis (scores 2-3) have significantly higher right atrial pressure than those with no fibrosis (score 0) or those with central fibrosis only (score 1, Figure 4b), suggesting that portal fibrosis is an indicator of more advanced disease. Because chronic right heart pressure or volume overload induces chamber dilatation, we assessed the relationship between the congestive hepatic fibrosis score and the severity of

Table 2 The demographic data and heart disease status

\begin{tabular}{|c|c|c|c|c|c|c|}
\hline & \multicolumn{5}{|c|}{ Congestive hepatic fibrosis score } & \multirow[b]{2}{*}{ P-values } \\
\hline & 0 & 1 & $2 A$ & $2 B$ & 3 & \\
\hline$N$ & 6 & 11 & 5 & 4 & 16 & \\
\hline Age & $58.8 \pm 1.5$ & $49.4 \pm 3.5$ & $47.6 \pm 7.6$ & $58.8 \pm 2.6$ & $50.3 \pm 3.1$ & $P=0.4$ \\
\hline Male (\%) & 66.7 & 81.8 & 80.0 & 75.0 & 56.3 & $P=0.7$ \\
\hline \multicolumn{7}{|l|}{ Laboratory data } \\
\hline AST & $50.3 \pm 16.7$ & $46.4 \pm 14.3$ & $30.8 \pm 4.6$ & $39 \pm 12.4$ & $33.1 \pm 3.1$ & $P=0.7$ \\
\hline ALT & $53.2 \pm 18.8$ & $87.8 \pm 62.1$ & $23 \pm 5.1$ & $29.3 \pm 7.8$ & $24.1 \pm 1.6$ & $P=0.67$ \\
\hline ALP & $75.7 \pm 5.8$ & $149.2 \pm 43.8$ & $111 \pm 21.8$ & $298 \pm 160$ & $149 \pm 29$ & $P=0.2$ \\
\hline GGT & $119 \pm 40$ & $136 \pm 42$ & $262 \pm 157$ & $151 \pm 47$ & $127 \pm 15$ & $P=0.5$ \\
\hline BIL-total & $0.8 \pm 0.2$ & $5.3 \pm 3.1$ & $2.4 \pm 0.4$ & $1.2 \pm 0.1$ & $2.5 \pm 0.4$ & $P=0.5$ \\
\hline BIL-direct & $0.2 \pm 0.1$ & $2.9 \pm 2.0$ & $0.8 \pm 0.3$ & $0.5 \pm 0.1$ & $1.1 \pm 0.3$ & $P=0.6$ \\
\hline \multicolumn{7}{|l|}{ Reason for biopsy } \\
\hline Preheart tx & 3 & 4 & 2 & 2 & 11 & \\
\hline Elevated LFT & 2 & 4 & 1 & 1 & 2 & \\
\hline Abnormal imaging & 1 & 3 & 2 & 1 & 3 & $P=0.77$ \\
\hline LV ejection fraction & $35 \pm 8.3$ & $25 \pm 6.4$ & $39.9 \pm 7.9$ & $28.5 \pm 7.5$ & $38.7 \pm 5.3$ & $P=0.87$ \\
\hline \multicolumn{7}{|c|}{ Heart disease classification } \\
\hline Idiopathic CM & 4 & 4 & 1 & 1 & 6 & \\
\hline Congenital HD & 0 & 3 & 1 & 0 & 6 & \\
\hline Ischemic CM & 1 & 3 & 0 & 1 & 2 & \\
\hline Valvular HD & 1 & 0 & 1 & 1 & 1 & \\
\hline Others & 0 & 1 & 2 & 1 & 1 & $P=0.46$ \\
\hline
\end{tabular}

Abbreviations: CM: cardiomyopathy; HD: heart disease; others: no specific etiology identified. 

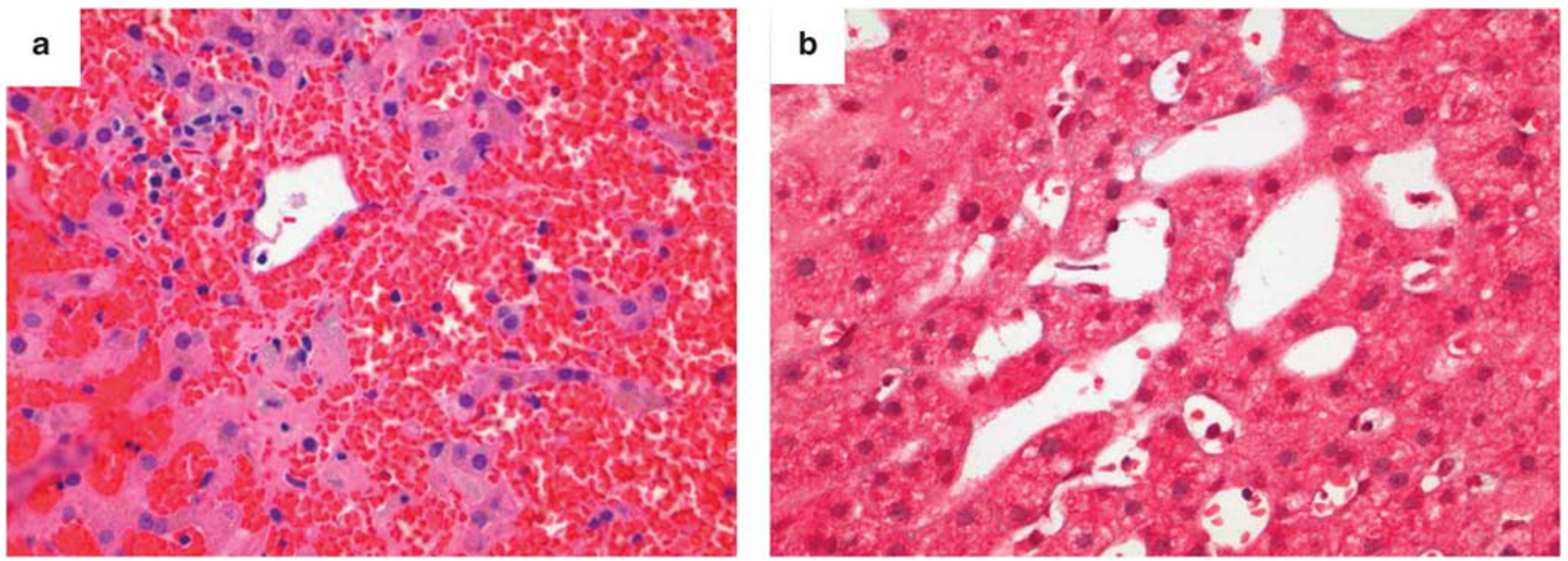

Figure 3 Moderate sinusoidal dilatation and hepatocyte atrophy in a patient with elevated right atrial pressure but no significant fibrosis (congestive hepatic fibrosis score 0). (a) Hematoxylin and eosin stain, high magnification; (b) Masson trichrome stain, high magnification.

Table 3 Congestive hepatic fibrosis score and frequencies of histologic findings

\begin{tabular}{|c|c|c|c|c|c|c|}
\hline & \multicolumn{5}{|c|}{$\begin{array}{l}\text { Congestive hepatic } \\
\text { fibrosis score }\end{array}$} & \multirow[b]{2}{*}{ P-values } \\
\hline & 0 & 1 & $2 A$ & $2 B$ & 3 & \\
\hline$N$ & 6 & 11 & 5 & 4 & 16 & \\
\hline Hepatocyte atrophy (\%) & 16.6 & 90.9 & 80.0 & 50.0 & 100.0 & $P<0.001$ \\
\hline \multicolumn{7}{|c|}{ Sinusoidal dilatation } \\
\hline Normal (\%) & 50.0 & 0.0 & 0.0 & 0.0 & 6.3 & \\
\hline Mild (\%) & 16.7 & 18.2 & 0.0 & 50.0 & 12.5 & \\
\hline Moderate (\%) & 33.3 & 54.5 & 100.0 & 50.0 & 62.5 & \\
\hline Severe $(\%)$ & 0.0 & 27.3 & 0.0 & 0.0 & 18.8 & $P=0.13$ \\
\hline
\end{tabular}

right atrial and ventricular dilation. As shown in Figures $5 \mathrm{a}$ and $\mathrm{b}$, increasing congestive hepatic fibrosis score is significantly associated with more severe right atrial and right ventricular dilatation.

As right heart catheterization is the gold standard for direct measurement of right atrial pressure, we compared the mean right atrial pressure obtained by right heart catheterization and pressure estimation by echocardiography using data available from 18 patients. There was no significant difference between RA pressure by right heart catheter measurement and RA pressure estimation by echocardiography, with a mean difference of $0.33 \pm$ $1.08 \mathrm{~mm} \mathrm{Hg}$.

\section{Discussion}

In this study we introduce a histologic fibrosis grading system for patients with congestive hepatopathy. We observe that sinusoidal dilatation, centrilobular hepatocyte atrophy, centrilobular fibrosis, and portal fibrosis correlate with right atrial pressure, whereas higher congestive hepatic fibrosis
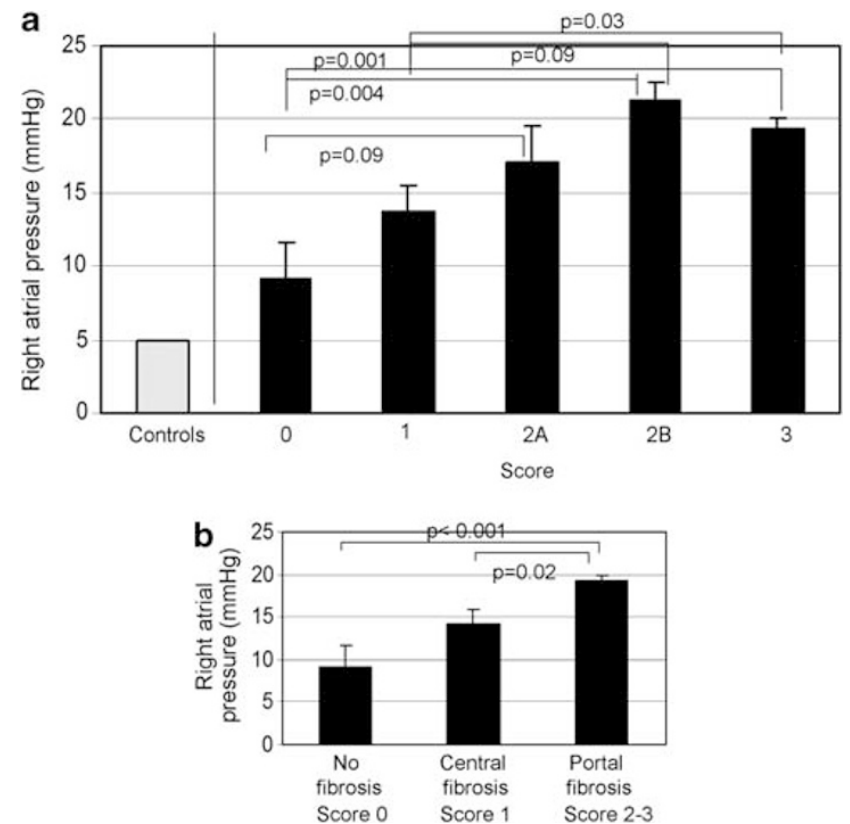

Figure 4 (a) Increased right atrial pressure ( $\mathrm{mm} \mathrm{Hg}$ ) with higher congestive hepatic fibrosis score, $P$ for trend $<0.001$. (b) Patients with portal fibrosis (scores 2-3) have significantly higher right atrial pressure than patients with no fibrosis (score 0) or central fibrosis only (score 1).

scores are associated with right atrial and right ventricular dilation.

Congestive hepatopathy is a common condition associated with chronic elevation of right heart pressure, commonly seen in end-stage heart failure or after Fontan operation. In patients with severe heart failure, particularly right heart failure, hepatic injury results from chronic congestion, with or without superimposed decline in hepatic arterial perfusion. ${ }^{6}$ Although this pathologic entity has been well known for decades, there has been no useful grading system for prospective and comparative 

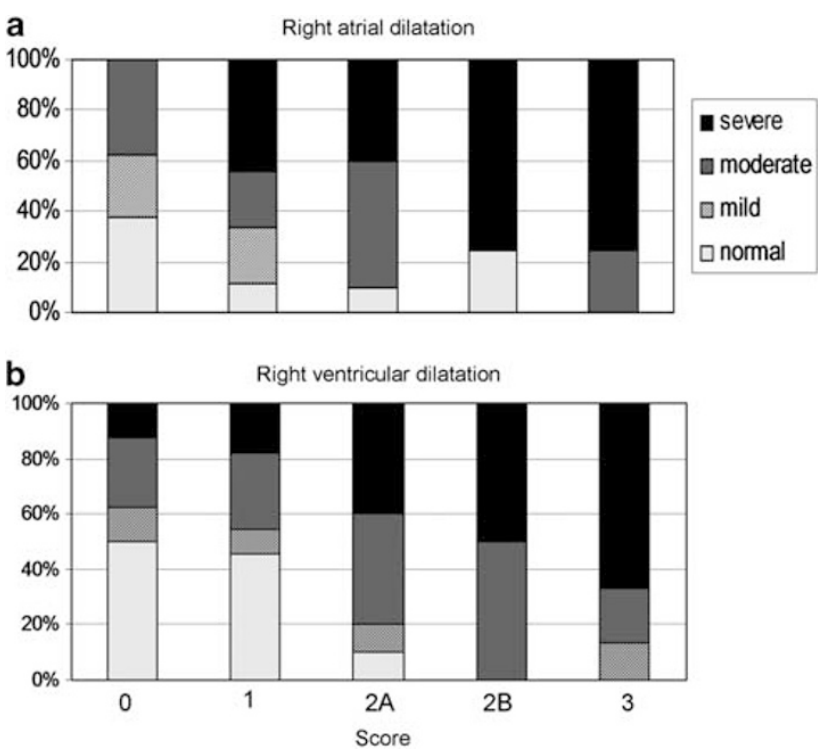

Figure 5 Proportions of various degrees of (a) right atrial dilatation and (b) right ventricular dilatation stratified by congestive hepatic fibrosis score. Higher score is significantly associated with greater degree of right atrial $(P=0.033)$ and ventricular $(P=0.024)$ dilatation.

studies, resulting in a lack of consistent reporting parameters for liver histopathology that potentially hinders the communication of appropriate information to the clinicians.

In this study, we have proposed a simple scoring system-the congestive hepatic fibrosis score-and have demonstrated that this score correlates well with echocardiography-derived right atrial pressure as well as both right atrial and right ventricular dilatation. Furthermore, the presence of portal fibrosis is associated with significantly higher right atrial pressure when compared with those without portal fibrosis (Figure 4b). As shown in Figure 1, the congestive hepatic fibrosis score is simple and straightforward and the above findings support the validity of this score as a useful indicator of the severity of congestive hepatopathy. The application of the congestive hepatic fibrosis score in this study was also a test of interobserver agreement. The initial independent evaluation of test cases by two pathologists assessed the degree of agreement (in this setting, $62.5 \%$ ) between one who developed the scoring system for the purposes of this study and one who independently reviewed the test material with reference only to the written scoring criteria without a training histologic set or representative images. After review of representative images (including those used in Figures 1 and 2) and a discussion of scoring criteria in the context of representative histologic material, the interobserver agreement reached $92.8 \%$. This emphasizes the role of appropriate training before employing this (or any other) histologic scoring system for prospective or retrospective studies.

Several classical autopsy studies have reported common histologic findings in post-mortem liver specimens from patients with cardiac diseases, including centrilobular congestion, centrilobular necrosis, inflammation, sclerosis, and regenerative hyperplasia. ${ }^{2,3}$ The evaluation of histopathology in autopsy, however, is often problematic because of various degrees of post-mortem autolysis and preservation artifacts. A recent study by Myers et $a l^{5}$ reported the clinical, hemodynamic, and histological features of liver biopsies from 83 patients with acute, acute on chronic, or chronic heart failure. They reported an association between free hepatic venous pressure and the presence of centrilobular necrosis, inflammation, periportal necrosis, and stainable hepatic iron, but not centrilobular fibrosis. Sinusoidal dilatation was also associated with higher right atrial and free hepatic venous pressures. ${ }^{5}$ It is noteworthy that the aforementioned studies included a heterogeneous patient cohort with various cardiac diseases and shock. In a large autopsy series of 1000 subjects with cardiac dysfunction, Arcidi et $a l^{3}$ reported that hepatic centrilobular necrosis often occurred in the setting of shock, whereas elevated central venous pressure often manifested as centrilobular congestion. These findings suggest that shock-induced liver injury and congestive hepatopathy are two different disease entities that might be best analyzed separately, as the former predominantly induces hypoxic cellular injury and necrosis, ${ }^{7}$ whereas the latter more likely involves stretch/pressure induced cell injury.

In patients with complex congenital heart disease results in a single functional ventricle (including hypoplastic left or right heart syndrome, and tricuspid or pulmonary atresia), the Fontan procedure bypasses the right ventricle via either a right atrium or central venous to pulmonary artery anastomosis. This results in chronic central venous hypertension, ${ }^{8}$ often $>20 \mathrm{~mm} \mathrm{Hg}$, and places patients at higher risk for cirrhosis. ${ }^{9}$ Kendall et al ${ }^{10}$ reported the results of liver biopsies from 18 patients after undergoing the Fontan procedure: all showed marked sinusoidal dilatation and mild ductular reaction; almost all had at least some degree of fibrosis, including bridging fibrosis in 14 patients and advanced-stage disease (cirrhosis) in 2 patients. Given the growing number of hepatocellular carcinoma cases in patients with congestive hepatopathy, a good scoring system is necessary for reporting consistency. Furthermore, congestive hepatic fibrosis score might be helpful to identify high-risk patients who need closer follow-up, screening for hepatocellular carcinoma, and consideration of heart versus heart and liver (dual organ) transplantation.

Congestive hepatic fibrosis increases the risk of postsurgical mortality in heart transplant recipients. ${ }^{11,12}$ Therefore, the American Heart Association Committee on Heart Failure and Cardiac Transplantation recommends that patients with coagulation abnormalities and aminotransferase levels higher than twice normal values should not be listed for heart transplant until the presence of severe liver 
disease has been excluded or the option of heart and liver transplant is deemed feasible. ${ }^{13}$ Further study is needed to elucidate whether congestive hepatic fibrosis score is useful for preoperative risk stratification to predict coagulopathy or hepatic decompensation in congestive hepatic fibrosis patients receiving major surgery.

Emerging evidence suggests that hepatic fibrosis in chronic liver disease may regress following removal of these insults. ${ }^{14}$ However, there is only one case report supporting the reversibility of congestive hepatic fibrosis after normalization of increased right heart pressure following heart transplantation. ${ }^{15}$ The clinical study of Dichtl et $a 1^{16}$ comprising 56 adult patients receiving heart transplantation indicated that many of the elevated liver tests significantly improve within 3 months after heart transplantation. ${ }^{16}$ In this setting, congestive hepatic fibrosis score will be useful to assess the reversibility of hepatic fibrosis following cardiac transplantation.

Previous reports on pathology of congestive hepatopathy have emphasized rheumatic heart disease and ischemic cardiomyopathy. Our present study included a diverse group of cardiac diseases, including idiopathic cardiomyopathy (38\%), congenital heart disease $(24 \%)$, ischemic cardiomyopathy $(17 \%)$, and valvular heart disease $(10 \%)$. It is important to note that neither the various etiologies of heart failure nor the left ventricular ejection fraction seem to correlate with the congestive hepatic fibrosis score, although this study may not have adequate sample size to determine whether different etiologies of heart failure correlate with the congestive hepatic fibrosis score. Nevertheless, our histopathologic observation demonstrates that sinusoidal dilatation, centrilobular hepatocyte atrophy, centrilobular fibrosis, and portal fibrosis more likely result from increased right atrial pressure because of the various causes of right heart failure.

A limitation of this study is the lack of diseasefree survival analysis, an element that limits the utility of the congestive hepatic fibrosis score for prognosis. This was not performed for two reasons: first, the progression of congestive hepatopathy to advanced cardiac cirrhosis is relatively rare, despite frequent manifestation of centilobular fibrosis, ${ }^{2,5}$ as discussed above; second, many of the patients in our study died of end-stage heart failure before the development of cardiac cirrhosis. With the strong confounding effect of heart failure and the lack of proper end point, the prognostic significance of the congestive hepatic fibrosis thus remains unknown. Despite this limitation, our data support the utilization of congestive hepatic fibrosis score as an indicator of disease severity.

In conclusion, our study has introduced a novel and simple histologic grading of hepatic fibrosis based on trichrome staining. This congestive hepatic fibrosis score is well correlated with the hemodynamic and echocardiographic parameters that reflect the severity of heart disease, supporting its utility as a valuable indicator of disease severity.

\section{Disclosure/conflict of interest}

The authors declare no conflict of interest.

\section{References}

1 Sherlock S. The liver in heart failure; relation of anatomical, functional, and circulatory changes. Br Heart J 1951;13:273-293.

2 Lefkowitch JH, Mendez L. Morphologic features of hepatic injury in cardiac disease and shock. J Hepatol 1986;2:313-327.

3 Arcidi JM Jr, Moore GW, Hutchins GM. Hepatic morphology in cardiac dysfunction: a clinicopathologic study of 1000 subjects at autopsy. Am J Pathol 1981;104:159-166.

4 Wanless IR, Liu JJ, Butany J. Role of thrombosis in the pathogenesis of congestive hepatic fibrosis (cardiac cirrhosis). Hepatology 1995;21:1232-1237.

5 Myers RP, Cerini R, Sayegh R, et al. Cardiac hepatopathy: clinical, hemodynamic, and histologic characteristics and correlations. Hepatology 2003;37:393-400.

6 Megalla S, Holtzman D, Aronow WS, et al. Predictors of cardiac hepatopathy in patients with right heart failure. Med Sci Monit 2011;17:CR537-CR541.

7 de la Monte SM, Arcidi JM, Moore GW, et al. Midzonal necrosis as a pattern of hepatocellular injury after shock. Gastroenterology 1984;86:627-631.

8 Ghaferi AA, Hutchins GM. Progression of liver pathology in patients undergoing the Fontan procedure: chronic passive congestion, cardiac cirrhosis, hepatic adenoma, and hepatocellular carcinoma. J Thorac Cardiovasc Surg 2005;129:1348-1352.

9 Krieger EV, Moko LE, Wu F, et al. Single ventricle anatomy is associated with increased frequency of nonalcoholic cirrhosis. Int J Cardiol 2013;167:1918-1923.

10 Kendall TJ, Stedman B, Hacking N, et al. Hepatic fibrosis and cirrhosis in the Fontan circulation: a detailed morphological study. J Clin Pathol 2008;61:504-508.

11 Assenza GE, Graham DA, Landzberg MJ, et al. MELDXI score and cardiac mortality or transplantation in patients after Fontan surgery. Heart 2013;99:491-496.

12 Chokshi A, Cheema FH, Schaefle KJ, et al. Hepatic dysfunction and survival after orthotopic heart transplantation: application of the MELD scoring system for outcome prediction. J Heart Lung Transplant 2012;31: 591-600.

13 Costanzo MR, Augustine S, Bourge R, et al. Selection and treatment of candidates for heart transplantation. A statement for health professionals from the Committee on Heart Failure and Cardiac Transplantation of the Council on Clinical Cardiology, American Heart Association. Circulation 1995;92:3593-3612.

14 Friedman SL, Bansal MB. Reversal of hepatic fibrosis fact or fantasy? Hepatology 2006;43:S82-S88.

15 Crespo-Leiro MG, Robles O, Paniagua MJ, et al. Reversal of cardiac cirrhosis following orthotopic heart transplantation. Am J Transplant 2008;8:1336-1339.

16 Dichtl W, Vogel W, Dunst KM, et al. Cardiac hepatopathy before and after heart transplantation. Transpl Int 2005;18:697-702. 